\title{
ON CERTAIN SUBCLASSES OF MEROMORPHICALIY MULTIVALENT FUNCTIONS
}

\author{
NAK EUN CHO
}

\begin{abstract}
The object of the present paper is to introduce a new class $J_{n, p}(\alpha)$ of meromorphically multivalent functions defined by a multiplier tranformation and to investigate some properties for the the class $J_{n, p}(\alpha)$. Our results include or improve some known results.
\end{abstract}

\section{Introduction}

Let $\sum_{p}$ denote the class of functions of the form

$$
f(z)=\frac{a_{-p}}{z^{p}}+\sum_{k=0}^{\infty} a_{k} z^{k} \quad\left(a_{-p} \neq 0, p \in N=\{1,2, \ldots\}\right)
$$

which are regular in the punctured disk $D=\{z: 0<|z|<1\}$. For any integer $n$, let the operator $I^{n}$ operating on $f \in \sum_{p}$ be defined by

$$
I^{n} f(z)=\frac{a_{-p}}{z^{p}}+\sum_{k=1}^{\infty}(p+k)^{-n} a_{k-1} z^{k-1}
$$

Obviously, we have

$$
I^{n}\left(I^{m}(f(z))=I^{n+m} f(z)\right.
$$

for all integers $m$ and $n$. For any nonpositive integer $n$ and $p=1$, the operators $I^{n}$ are the differential operators studied by Uralegaddi and Somanatha [6,7]. Also the operators $I^{n}$ are closely related to the multiplier transformations introduced by Flett [2].

For any integer $n$, let $J_{n, p}(\alpha)$ denote the class of functions $f \in \Sigma_{p}$ satisfying the condition

$$
\operatorname{Re}\left\{\frac{\left(I^{n-1} f(z)\right)^{\prime}}{\left(I^{n} f(z)\right)^{\prime}}-(p+1)\right\}<-\alpha \quad(0 \leq \alpha<p, z \in U=\{z:|z|<1\}) .
$$

Received February 18, 1994; revised June 15, 1994.

1991 Mathematics Subject Classification. 30C45.

Key words and phrases. Meromorphically $p$-valent convex functions of order $\alpha$, integral operators. 
In this paper, it is shown that the integral operator $F_{c}$ defined by

$$
F_{c}(z)=\frac{c}{z^{c+p}} \int_{0}^{z} t^{c+p-1} f(t) d t \quad(c \geq 1)
$$

belongs to the class $J_{n, p}(\alpha)$, whenever $f \in J_{n, p}(\alpha)$. From this result, we also prove that for the classes $J_{n, p}(\alpha)$ of functions in $\sum_{p}, J_{n, p}(\alpha) \subset J_{n+1, p}(\alpha)$ holds. Since $J_{0, p}(\alpha)$ equals to the class of meromorphically p-valent convex functions of order $\alpha$, all members in $J_{n, p}(\alpha)$ are p-valent convex for any nonpostive integer $n$ [4]. Our results generralize some results of Bajpai [1], Goel and Sohi [3].

\section{Main results}

We begin with the statement of the following lemma due to Miller and Mocaun [5].

Lemma. Let $\phi(u, v)$ be a complex valued function, $\phi: R \rightarrow \mathbb{C}, R \subset \mathbb{C}^{2}(\mathbb{C}$ is the complex plane), and let $u=u_{1}+i u_{2}, v=v_{1}+i v_{2}$. Suppose that the function $\phi(u, v)$ satisfies the following condition:

(i) $\phi(u, v)$ is continuous in $R$;

(ii) $(1,0) \in R$ and $\operatorname{Re}\{\phi(1,0)\}>0$;

(iii) $\operatorname{Re}\left\{\phi\left(i u_{2}, v_{1}\right)\right\} \leq 0$ for all $\left(i u_{2}, v_{1}\right) \in R$ such that $v_{1} \leq \frac{-\left(1+u_{2}^{2}\right)}{2}$ Let $r(z)=1+r_{1} z+r_{2} z^{2}+\cdots$ be regular in $U$ such that $\left(r(z), z r^{\prime}(z)\right) \in R$ for all $z \in U$. If

$$
\operatorname{Re}\left\{\phi\left(r(z), z r^{\prime}(z)\right)\right\}>0 \quad(z \in U),
$$

then $\operatorname{Re}\{r(z)\}>0(z \in U)$.

With the aid of above lemma, we derive

Theorem 1. Let $f \in J_{n, p}(\alpha)$ and let

$$
F_{c}(z)=\frac{c}{z^{c+p}} \int_{0}^{z} t^{c+p-1} f(t) d t \quad(c \geq 1) .
$$

Then $F_{c} \in J_{n, p}(\beta)$, where

$$
\beta=\frac{2(p+\alpha)+2 c+1-\sqrt{(2(p-\alpha)-2 c+3)^{2}+8(2(p+1-\alpha)(c-1)+1)}}{4} .
$$

Proof. Let $f \in J_{n, p}(\alpha)$. Then we have

$$
\operatorname{Re}\left\{\frac{\left(I^{n-1} f(z)\right)^{\prime}}{\left(I^{n} f(z)\right)^{\prime}}-(p+1)\right\}<-\alpha .
$$


From the definition of $F_{c}$, we obtain

$$
z\left(I^{n} F_{c}(z)\right)^{\prime}=c I^{n} f(z)-(c+p) I^{n} F_{c}(z)
$$

and also

$$
z\left(I^{n} F_{c}(z)\right)^{\prime}=I^{n-1} F_{c}(z)-(p+1) I^{n} F_{c}(z) .
$$

Using (2.4) and (2.5), the condition (2.3) may be written as

$$
\operatorname{Re}\left\{\frac{\frac{\left(I^{n-2} F_{c}(z)\right)^{\prime}}{\left(I^{n-1} F_{c}(z)\right)^{\prime}}+(c-1)}{1+(c-1) \frac{\left(I^{n} F_{c}(z)\right)^{\prime}}{\left(I^{n-1} F_{c}(z)\right)^{\prime}}}-(p+1)\right\}<-\alpha .
$$

Define the function $r(z)$ by

$$
\frac{\left(I^{n-1} F_{c}(z)\right)^{\prime}}{\left(I^{n} F_{c}(z)\right)^{\prime}}=\gamma+(1-\gamma) r(z)
$$

where

$$
\gamma=\frac{2(p-\alpha)-2 c+3+\sqrt{(2(p-\alpha)-2 c+3)^{2}+8(2(p+1-\alpha)(c-1)+1)}}{4} \quad(\gamma>1) .
$$

Then $r(z)=1+r_{1} z+r_{2} z^{2}+\cdots$ is regular in $U$. Differentiating (2.7) logarithmically and simplifying, we have

$$
\begin{aligned}
\frac{\frac{\left(I^{n-2} F_{c}(z)\right)^{\prime}}{\left(I^{n-1} F_{c}(z)\right)^{\prime}}+(c-1)}{1+(c-1) \frac{\left(I^{n} F_{c}(z)\right)^{\prime}}{\left(I^{n-1} F_{c}(z)\right)^{\prime}}}-(p+1)= & -(p+1)+\gamma+(1-\gamma) r(z) \\
& +\frac{(1-\gamma) z r^{\prime}(z)}{(\gamma+c-1)+(1-\gamma) r(z)}
\end{aligned}
$$

It follows from (2.9) that

$$
\begin{aligned}
& -\operatorname{Re}\left\{\frac{\frac{\left(I^{n-2} F_{c}(z)\right)^{\prime}}{\left(I^{n-1} F_{c}(z)\right)^{\prime}}+(c-1)}{1+(c-1) \frac{\left(I^{n} F_{c}(z)\right)^{\prime}}{\left(I^{n-1} F_{c}(z)\right)^{\prime}}}-(p+1)+\alpha\right\} \\
= & \operatorname{Re}\left\{p+1-(\alpha+\gamma)-(1-\gamma) r(z)-\frac{(1-\gamma) z \gamma^{\prime}(z)}{(\gamma+c-1)+(1-\gamma) r(z)}\right\} \\
> & 0 .
\end{aligned}
$$

If we define the function $\phi(u, v)$ by

$$
\phi(u, v)=p+1-(\alpha+\gamma)-(1-\gamma) u-\frac{(1-\gamma) v}{(\gamma+c-1)+(1-\gamma) u}
$$


then $\phi(u, v)$ satisfies

(i) $\phi(u, v)$ is continuous in $R=\left(\mathbb{C}-\left\{\frac{\gamma+c-1}{\gamma-1}\right\}\right) \times \mathbb{C}$;

(ii) $(1,0) \in R$ and $\operatorname{Re}\{\phi(1,0)\}=p-\alpha>0$;

(iii) for all $\left(i u_{2}, v_{1}\right) \in R$ such that $v_{1} \leq \frac{-\left(1+u_{2}^{2}\right)}{2}$,

$$
\begin{aligned}
\operatorname{Re}\left\{\phi\left(i u_{2}, v_{1}\right)\right\} & =p+1-(\alpha+\gamma)-\frac{(\gamma+c-1)(1-\gamma) v_{1}}{(\gamma+c-1)^{2}+(1-\gamma)^{2} u_{2}^{2}} \\
& \leq p+1-(\alpha+\gamma)+\frac{(\gamma+c-1)(1-\gamma)\left(1+u_{2}^{2}\right)}{2\left\{(\gamma+c-1)^{2}+(1-\gamma)^{2} u_{2}^{2}\right\}} \\
& \leq 0
\end{aligned}
$$

Since $\phi(u, v)$ satisfies the conditions in Lemma, we have that $\operatorname{Re}\{r(z)\}>0(z \in U)$. This proves that

$$
\operatorname{Re}\left\{\frac{\left(I^{n-1} F_{c}(z)\right)^{\prime}}{\left(I^{n} F_{c}(z)\right)^{\prime}}\right\}<\gamma \quad(z \in U)
$$

or

$$
\operatorname{Re}\left\{\frac{\left(I^{n-1} F_{c}(z)\right)^{\prime}}{\left(I^{n} F_{c}(z)\right)^{\prime}}-(p+1)\right\}<-\beta \quad(z \in U, 0 \leq \beta<p),
$$

where $\beta$ is given by (2.2). That is, $F_{c} \in J_{n, p}(\beta)$.

Since $\beta-\alpha>0$ in Theorem 1 , we have

Corollary 1. If $f \in J_{n, p}(\alpha)$, then the integral operator $F_{c}$ defined by (2.1) belongs to the class $J_{n, p}(\alpha)$.

Taking $n=0, p=1$ and $\alpha=0$ in Corollary 1 , we obtain the following corresponding result of Goel and Sohi [3].

Corollary 2. If $f(z)=\frac{a-1}{z}+\sum_{k=0}^{\infty} a_{k} z^{k}\left(a_{-1} \neq 0\right)$ is meromorphically convex, than so is the integral operator $F_{c}$ defined by (2.1).

Putting $c=1$ in Corollary 2, we obtain the following result of Bajpai [1].

Corollary 3. If $f(z)=\frac{a-1}{z}+\sum_{k=0}^{\infty} a_{k} z^{k}\left(a_{-1} \neq 0\right)$ is meromorphically convex, then so is

$$
F_{1}(z)=\frac{1}{z^{2}} \int_{0}^{z} t f(t) d t
$$

Next, we prove

Theorem 2. If $f \in J_{n, p}(\alpha)$, then $f \in J_{n+1, p}(\beta)$, where

$$
\beta=\frac{3+2(p+\alpha)-\sqrt{(2(p-\alpha)+1)^{2}+8}}{4} .
$$


Proof. For $c=1$, the identities (2.4) and (2.5) reduce to $I^{n} f(z)=I^{n-1} F_{1}(z)$ and hence $I^{n+1} f(z)=I^{n} F_{1}(z)$. Therefore

$$
\frac{\left(I^{n} f(z)\right)^{\prime}}{\left(I^{n+1} f(z)\right)^{\prime}}=\frac{\left(I^{n-1} F_{1}(z)\right)^{\prime}}{\left(I^{n} F_{1}(z)\right)^{\prime}}
$$

Since $f \in J_{n, p}(\alpha)$, the result follows from Theorem 1 .

Similarly, from Theorem 2, we have

Corollary 4. $J_{n, p}(\alpha) \subset J_{n+1, p}(\alpha)$ for any integer $\dot{n}$.

Remark. Since $J_{0, p}(\alpha)$ is the class of meromorphically $p$-valent convex functions of order $\alpha$ [4], we can see from Corollary 3 that all members in $J_{n, p}(\alpha)$ are meromorphically $p$-valent convex of order $\alpha$ for any nonpostive integer $n$.

We state the following theorem which is proved by a similar method of Theorem 2 .

Theorem 3. $f \in J_{n, p}(\alpha)$ if and only if the integral operator $F_{1}$ defined by (2.16) belongs to the class $J_{n-1, p}(\alpha)$.

Acknowledgements. The author is thankful to the referee for his helpful comments. This paper was supported (in part) by Non Directed Research Fund and the Research Institute Attached to University Program, Korea Research Foundation, 1993.

\section{References}

[1] S. K. Bajpai, "A note on a class of meromorphic univalent functions," Rev. Roumanie Math. Pure Appl., 22(1977), 295-297.

[2] T. M. Flett, "The dual of an inequality of Hardy and Littlewood and some related inequalities," J. Math. Anal. Appl., 38(1972), 746-765.

[3] R. M. Goel and N. S. Sohi, "On a class of meromophic functions," Glas. Mat., 17(1981), 19-28.

[4] V. Kumar and S. C. Shukla, "Certain integrals for classes of p-valent meromorphic functions," Bull. Austral. Math. Soc., 25(1982), 85-97.

[5] S. S. Miller and P. T. Mocanu, "Second order differential inequalities in the complex plane," J. Math. Anal. Appl., 65(1978), 289-305.

[6] B. A. Uralegaddi and C. Somanatha, "Certain differential operators for meromorphic functions," Houston J. Math., 17(1991), 279-284.

[7] B. A. Uralegaddi and C. Somanatha, "New criteria for meromorphic starlike univalent functions," Bull. Austral. Math. Soc., 43(1991), 137-140. 